\title{
Mechanisms Generating Asymmetric Core-Cohesive Blockmodels
}

\author{
Marjan Cugmas ${ }^{1}$
}

\author{
Aleš Žiberna ${ }^{2}$
}

Anuška Ferligoj ${ }^{3}$

\begin{abstract}
The paper addresses the relationship between different local network mechanisms and different global network structures, described by blockmodels. The research question is narrowed to the context of preschool children networks. Based on the studies regarding friendship, liking and interactional networks among preschool children, the popularity, transitivity, mutuality and assortativity mechanisms are assumed to be important for the evolution of such networks. It is assumed that the global network structure is defined by an asymmetric core-cohesive blockmodel consisting of one core group of units and two or more cohesive groups of units. Therefore, the main research question is whether the emergence of an asymmetric core-cohesive blockmodel can be a result of the influence of the listed mechanisms. Different initial global network structures are considered. Monte Carlo simulations were used. The relative fit measure is proposed and used to compare different blockmodel types on generated networks. The results show that the listed mechanisms indeed lead to the assumed global network structure.
\end{abstract}

\section{Introduction}

This paper discusses how different local network mechanisms affect a global network structure, which is described by a blockmodel. A blockmodel is defined as a network in which the nodes represent groups of equivalent units (according to their structure of relationships) and the links that represent relationships between and within the groups. The term block refers to a submatrix that shows the relationships between units of two groups or within one group. Typical blockmodel types are cohesive, core-periphery, hierarchical and transitive (Doreian, Batagelj and Ferligoj, 2005).

Knowing the relationship between micro social network mechanisms and global network structures is useful while analyzing empirical networks and it helps to select mechanisms when using the exponential random graph modelling (Hunter, Goodreau and Handcock, 2013) or stochastic actor-based modelling (Snijders, Van de Bunt and Steglich, 2010).

\footnotetext{
${ }^{1}$ Faculty of Social Sciences, University of Ljubljana, Ljubljana, Slovenia; marjan.cugmas@fdv.unilj.si

${ }^{2}$ Faculty of Social Sciences, University of Ljubljana, Ljubljana, Slovenia; ales.ziberna@fdv.uni-lj.si

${ }^{3}$ Faculty of Social Sciences, University of Ljubljana, Ljubljana, Slovenia; Higher School of Economics, National Research University, Moscow, Russia; anuska.ferligoj@fdv.uni-lj.si
} 
As there are many possible blockmodel types and many different local network mechanisms, the focus of the paper is narrowed on a specific blockmodel type and local network mechanisms, which are relevant for preschool liking, popularity and friendship networks.

When children enter to the kindergarten, they start forming groups. Children within such groups spend more time with each other than with those from other groups. At the same time, a group (popular group) of children is formed with whom all the others want to spend considerable amount of time. Therefore, the assumed blockmodel is the asymmetric core-cohesive blockmodel type, which is assumed to be present in empirical preschool children networks. This blockmodel type is a mixture of two very common blockmodel types, namely asymmetric core-periphery blockmodel type and cohesive blockmodel type (see Section 1.1).

Based on the studies regarding friendship, liking and interactional networks among preschool children, the popularity, transitivity, mutuality and assortativity mechanisms are assumed to be important for the evolution of such networks (see Section 1.2). Another reason for choosing the selected mechanisms is that the popularity mechanism leads toward the asymmetric core-periphery blockmodel type and the transitivity-related mechanism leads toward the cohesive blockmodel type. All of the mentioned mechanisms and the proposed blockmodel type are described in details below. The study is limited to binary networks.

Even though this paper is limited to only one particular global network structure and several well-known local network mechanisms, the proposed methodology can be applied to study other local network mechanisms and global network structures. Of course, the global network structures and the local mechanisms should be chosen within a specific context of a study.

\subsection{Core-Cohesive Blockmodel}

The core-cohesive blockmodel type consists of at least three groups of units. The units of each group are internally well linked. All units are linked to the core group of units. Such a blockmodel type with three groups is graphically represented in Figure 1. Here, the nodes represent the groups of units. Group 1 consists of internally highly linked units and is called a core group. The units in cohesive groups are internally highly linked and are linked to the core group. The blockmodel may be extended in such a way that the units from one cohesive group would not be highly internally linked.

The core group can be named the popular group since the term "popularity" is often associated with the high in-degree, which is a characteristic of the core group of units. The term core is more general than the term popular.

\subsection{Mechanisms that Lead to a Core-Cohesive Blockmodel}

Because the social mechanisms are very content-specific, it is sometimes not trivial to select mechanisms that would drive a network towards a specific global structure. Since the core-cohesive global network structure has not been formally presented in the form of a blockmodel yet, it has not been mentioned in empirical studies concerning the evolution of global network structures. 

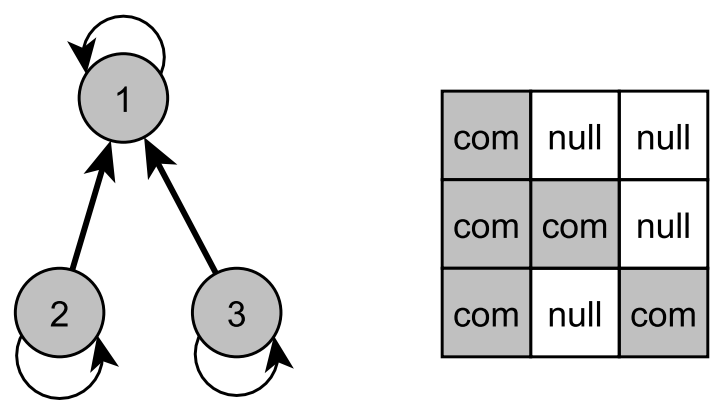

Figure 1: Graphic (left) and matrix (right) representation of the core-cohesive blockmodel with three groups

On the assumption the proposed blockmodel type is present in the liking friendship networks among the preschool children (or, more precisely, among children at kindergarten), several mechanisms that might lead towards the proposed blockmodel type can be identified. As shown later, the popularity and transitivity-related mechanisms have been extensively studied in this context. While the first one leads to the asymmetric coreperiphery blockmodel type, the second one is associated with the cohesive blockmodel type. At this point, it must be emphasised that the units' characteristics are not considered in this study, even though they can play an important role in the formation of links in real networks. $^{2}$

When evaluating studies of networks among preschool children in the context of a global network structure, one must differentiate between popularity and friendships. ${ }^{3}$ Popularity refers to how a group views an individual, according, according to liking and disliking, while friendship is conceptualised as a bilateral construct (although empirical friendship networks are usually asymmetric). Considering liking as being the basic link between popularity and friendship, Bukowski et al. (1996) showed that the positive association between popularity and friendships decreases with age.

Snyder et al. (1996) noticed that preschool children spend a lot of time with selected friends and less with others. Schaefer et al. (2010) studied three of the most common net-

\footnotetext{
${ }^{2}$ Kerns (2000) studied the friendships among children aged 42 to 84 months and confirmed the existence of several types of friendships (e.g., a cluster of harmo nious, responsive and interactive friendships and a cluster of harmonious but independent friendships). Some types are expected to last a longer time while others are expected to last a shorter period. However, also for the latter it can happen that they last relatively long, which may be a consequence of some personal characteristics, e.g., in some cases friendships can be the result of geographical space between two children or the result of lacking social skills of one or both children in a current frien dship. Similarly, Proulx and Poulin (2013) confirmed that personal characteristics such as aggressiveness, social pro - activeness and shyness affect the number and stability of friendships among children at kindergarten (also see Engle, McElwain and Lasky (2011)). Depending on the age of children, gender is also an important personal characteristic when forming cohesive groups of friends (Johnson et al., 1997). Adams and Torr (1998) highlighted that friendships are much more affected by the cultural context than any other institutionalised relationships.

${ }^{3}$ This was also considered when operationalising popularity through in-degree in empirical networks. For example, when thinking about friendships in a preschool environment the number of peer contacts relates more to the perception of popularity among peers than the quality of contacts. Those with a higher number of peer contacts and those who in the centre of the network are therefore seen as more popular while the isolates are seen as less popular by others (LaFontana and Cillessen 2002). Popularity can be achieved via positive or negative behaviour (Cillessen and Rose 2005).
} 
work formation mechanisms (reciprocity, popularity and triadic closure) among preschool children throughout a school year in four waves using SIENA (a program for statistically analysing network data based on stochastic actor-oriented models, see Snijders, Van de Bunt and Steglich (2010)). They found the reciprocity effect is constant over time while the importance of popularity is highest midway through the school year. The importance of the triadic closure effect increases in time, which is expected since very early friendships are typically play-oriented dyads that primarily socialise children into group life (Hartup and Stevens, 1997). When children gain more social contacts and greater confidence, they move into larger groups (Hartup, 1993).

Daniel et al. (2013) used Exponential Random Graph Models (ERGM) to study reciprocity, popularity and transitivity in relation to the formation of affiliative ties in 19 Portuguese preschool peer groups. ERGMs were estimated with the same network effects for all 19 classes. They found positive popularity and triadic closure effects which, as they reported, may indicate the existence of a core-periphery global network structure. Since they found some children with a very high in-degree, the true underlying global network structure might be close to core-cohesive.

\subsection{Research Question}

The study of the relationship between local network (social) mechanisms and the global network structure is based on the following research question: Can the selected mechanisms lead the network towards the core-cohesive blockmodel structure? The research question is then broken up into parts. Therefore, we consider the following sub-questions concerning whether a core-cohesive blockmodel can emerge: (i) from an empty network; (ii) from a cohesive blockmodel; or (iii) from an asymmetric core-periphery blockmodel. All of these cases consider popularity, assortativity, two transitivity-related mechanisms, outgoing shared partners and mutuality mechanisms as local network mechanisms.

\subsection{Structure of the Article}

This article is divided into several sections. The algorithm for generating the networks is proposed and described in Section 2.1 while the mechanisms used in this algorithm are formally defined in Section 2.2. Methods for evaluating the global network structures are presented in Section 2.4, with the results being given in Section 3. The latter is further organised in several subsections according to the initial networks' global network structure, followed by a section that discusses the ways in which different mechanisms simultaneously affect the global network structure.

\section{Methods}

Probability models have already been proposed for friendship networks, consisting of a given number of cliques (Jansson, 1997), and for the popularity structure in social networks, where popularity is defined by the in-degree (Jansson, 2000). Our analysis proposes and uses a more deterministic model from the family of network evolution models 
(NEM) (Toivonen, et al., 2009). The global network structure is tested by the relative value of the criterion function, called "relative fit" (RF).

The model imposes two main assumptions that are also common in the continuoustime Markov process: two relationships cannot simultaneously change and the probability of a link changing can be expressed as a function of the entire network at a certain point in time (Zeggelink, 1994). In our proposed network evolution model, the global network structure in the following step depends on the global network structure in the current step.

The units' actions are goal-oriented (Snijders, 1996), meaning that "each unit takes actions in order to fulfil his own goals; these actions are in the domain of his own behaviour or of the directed relationships from him to others". The actions are operationalised using selected mechanisms.

\subsection{Network Evolution Model}

Before applying the iterative algorithm for generating random networks (Algorithm 1), one must specify the initial network $X_{0}$ in the form of a binary $n \times n$ adjacency matrix $X$, the vector of the weights of the local mechanisms $\theta$, the probability of establishing a link at each iteration $q$ and the number of iterations $m$. The algorithm is as follows: at each iteration, unit $i$ is randomly selected with probability $1 / n$. Then, the network statistics $S$ are obtained based on the operationalised selected mechanisms presented in the next subsection. These network statistics are weighted by the vector of weights of local mechanisms $\theta$ producing vector $\varphi=S \theta^{T}$. These units, for which it holds $\varphi \geq$ $Q_{3}(\varphi)\left(Q_{3}\right.$ stands for the 3 rd quartile), are classified in set $\mathcal{C}$ and are candidates to accept the incoming tie from unit $i$. The other units, for which it holds $\varphi \leq Q_{1}(\varphi)\left(Q_{1}\right.$ stands for the 1st quartile), are classified in set $\mathcal{F}$ and are candidates for being dissolved by incoming tie by unit $i$. With probability $q$, the link from $i$ to randomly selected $j$ from set $\mathcal{C}$ is set and with the probability $1-q$ a non-link from $i$ to randomly selected $j$ from set $\mathcal{F}$ is established. The fact the unit can establish a link to already existing links can result in no visible change of a link upon a given iteration. The links in the resulting network are represented by $1 \mathrm{~s}$ while the non-links are represented by $0 \mathrm{~s}$.

\subsection{Local Mechanisms}

Different mechanisms can be considered in the proposed NEM. The term "mechanism" describes a process that drive the concrete actions by units in the networks (e.g., creating a link to a highly-popular unit). These mechanisms can be operationalised by different statistics (e.g., in the alter in-degree) that reflect the mechanisms.

As described in the previous section, the proposed network statistics $S$ are weighted by a given vector $\theta$. A higher positive weight is associated with the greater importance of a given mechanism while a lower positive weight is associated a given mechanism's reduced importance. Higher negative weights are also associated with a mechanism that is more important, but the interpretation of the mechanism's effect is different. For example, a negative weight for the popularity mechanisms is interpreted as a tendency to avoid creating links to highly popular others.

In the current study, the following mechanisms are defined and used: mutuality (M), assortativity (A), popularity (P), transitivity (T) and outgoing shared partners (OSP). They 


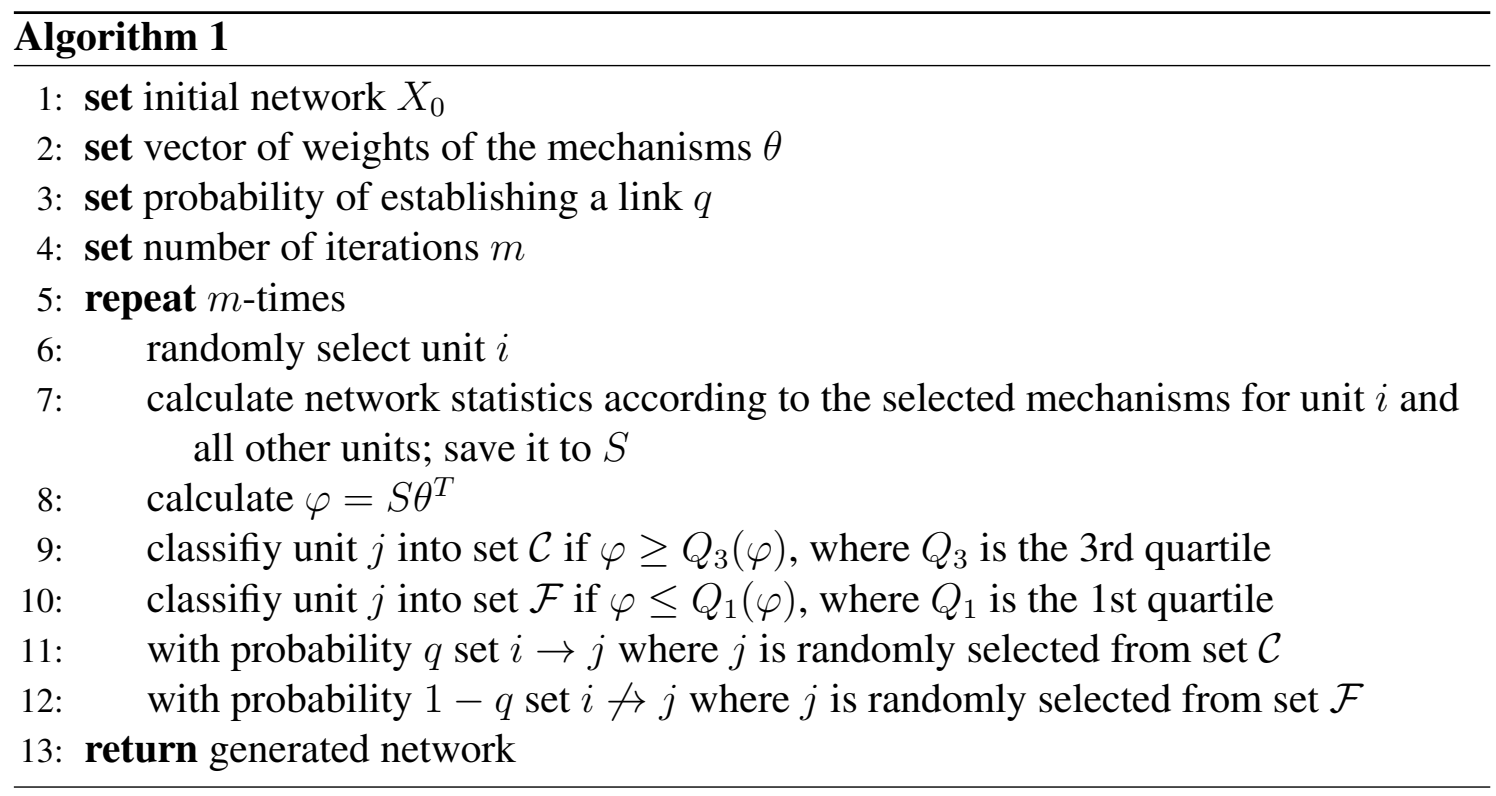

are defined on a network with $n$ units (nodes), represented by the binary $n \times n$ adjacency matrix $X$, and are normalised in such a way that the minimum corresponding values are 0 and the maximum values are 1 . The different mechanisms considered are schematically shown in Figure 2 where dashed lines illustrate the links under evaluation appear, are confirmed or disappear. The mechanism presented in Figure 2a corresponds to the tendency of having a link. Since this is not a focal mechanism, it is implemented in the NEM algorithm as parameter $q$. The following mechanisms are considered in this paper:

1. The mutuality mechanism (Figure $2 b$ ) reflects the tendency to reciprocate links. It is operationalised by asymmetric in-ties statistic as

$$
\operatorname{asymIn}(i, j)= \begin{cases}1, & \text { if } x_{i j}+(-1) \cdot x_{i j}=1 \\ 0, & \text { otherwise }\end{cases}
$$

The first condition would be satisfied only in the case when there would be a nonreciprocal link from unit $j$ to unit $i$. We are using normalised statistic $(M)$ defined as

$$
M(i, j)=\frac{\operatorname{asym} \operatorname{In}(i, j)}{\sum_{j=1}^{n} \operatorname{asym} \operatorname{In}(i, j)}
$$

2. The alter popularity mechanism (Figure 2c) (referred to as the "popularity mechanism" below) reflects the tendency of creating links to the most popular ones. The popularity statistic (P) is calculated for $i$-th unit as the ratio between the in-degree of the $i$-th unit and the total number of units

$$
P(i, j)=\frac{\sum_{j=1}^{n} x_{i j}}{n-1}
$$

3. The assortativity mechanism (Figure $2 \mathrm{~d}$ ) reflects the tendency to create links to those units with the same level of popularity (in-degree). Assortativity statistic (A) 
is defined as

$$
A(i, j)=\max \left(1-\frac{\left|\sum_{i=1}^{n} x_{i j}-\sum_{j=1}^{n} x_{i j}\right|^{2}}{4}, 0\right)
$$

and can be calculated for each pair of units: if $i$ and $j$ have the same in-degree, then they have the same assortativity with all other units. The function $A(i, j)$ has the value 1 when units $i$ and $j$ do not differ in the number of incoming ties. In our model, the unit that has a chance to change a tie is chosen and therefore for only this unit and all the others the function $A(i, j)$ is applied.

4. The transitivity-related mechanisms reflect the tendency to create links to those units with whom a unit shares a higher number of reciprocated or non-reciprocated links to other units in a network. The concept of the selected mechanisms generalises the ideas of triangles and 2-stars (Snijders, Pattison, Robins and Handcock, 2006). It is very common, especially when analysing the networks by ERGM (Robins, Pattison, Kalish and Lusher, 2007) or SIENA (Snijders, Van de Bunt, and Steglich, 2010). There are four types of shared partners (Robins et al. 2009), yet just two of them are considered in this paper:

- Transitivity (T) also referred to as an outgoing two-path (OTP) (Figure 2e): a transitivity relation is defined as $i \rightarrow j$ where also $i \rightarrow k \rightarrow j$. That is, transitivity is a tendency for a unit to directly connect to units to which it is indirectly connected with (one or more) paths of length two (with more paths increasing the tendency).

- Outgoing shared partner (OSP) (Figure 2f): unit $k$ is a shared partner of the ordered pair $(i, j)$ if $i \rightarrow k$ and $j \rightarrow k$; the OSP represents a "structural homophily" effect which is traditionally based on similarity according to the units' attributes. In the case of the OSP, it is defined by similar choices of partners (Robins, Pattison and Wang, 2009).

To compute the statistics associated with these mechanisms on the selected pair of units $i$ and $j$, one must identify the other units (not $i$ and not $j$ ) which are linked with $i$ and $j$ (shared partners) in a given way. When the transitivity statistic is considered, the number of alternative intermediaries on two paths from $i$ to $j$ are computed as

$$
T(i, j)=\sum_{k \neq i, j} x_{k i} \cdot x_{j k}
$$

Similarly, the number of outgoing shared partners is computed as

$$
O S P(i, j)=\sum_{k \neq i, j} x_{j k} \cdot x_{i k} .
$$

Both functions give the number of shared partners of a given type between $i$ and $j$. By fixing unit $i$, one can obtain vector $V$ with $n$ elements where each value stands for the number of common friends between the unit and all other units. The $i$-th 
value of vector $V$ can be normalised as $V_{i} / \sum_{i=1}^{n} V_{i}$. Such normalised statistics are used as the operationalisations of the transitivity and OSP mechanisms.

In this paper, it is assumed that the mechanisms' weights are the same for all units regardless of their position in the network. However, the position of a unit in a network indirectly influences others to whom a unit sets a tie or a non-tie. This is because the selected units are chosen based on the weighed network statistics $(\varphi)$. While the weights are the same for all units, the network statistics can vary among units in the network, depending on their position in the network. ${ }^{4}$

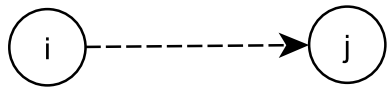

(a) parameter q

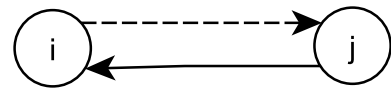

(b) mutuality

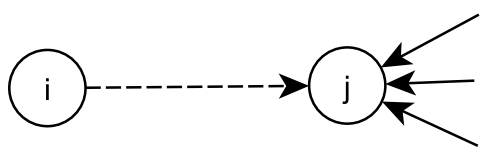

(c) popularity

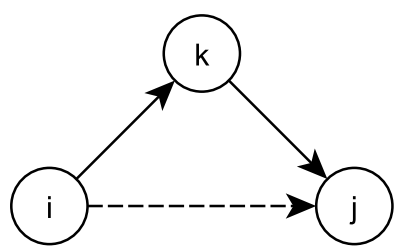

(e) transitivity

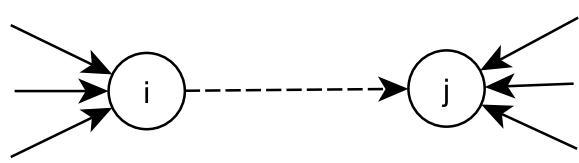

(d) assortativity

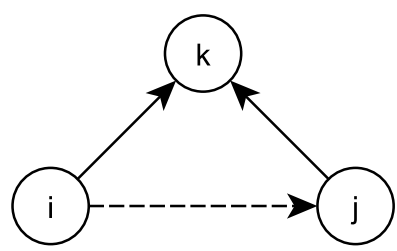

(f) outgoing shared partners

Figure 2: Illustrations of the different mechanisms considered

Preliminary analysis (not included in this paper) shows that considering only the popularity mechanism results in networks with the asymmetric core-periphery blockmodel type, while considering only the OSP mechanism results in networks with the cohesive blockmodel type. The global network structures of the generated networks when considering the mutuality and assortativity mechanisms do not end in any known blockmodel type (e.g., cohesive, core-periphery, transitivity, hierarchy).

\footnotetext{
${ }^{4}$ There are some other considerations, which are also common for most of the empirical studies that are using SIENA or ERGM. For example, the weights of the mechanisms are fixed for all units regardless of their attributes. In addition, a global network structure can influence the importance of the selected mechanisms. For example, some empirical studies on friendship networks show that the mutuality mechanism might be weaker for those units involved in transitive triplets, compared to the others (Block, 2015). However, differentiating between the strength of the impact of a global network structure to the set of mechanisms' strengths of a given unit and vice versa is hard and very context- specific.
} 


\subsection{Selecting the Weights of the Mechanisms}

One way to select the set of mechanisms' weights is to generate networks using the proposed model with different sets of the mechanism' weights (in this study, 30 networks were generated with each set of the mechanisms' weights). To access whether the generated networks have the asymmetric core-cohesive blockmodel structure, non-specified generalised blockmodeling (Doreian, Batagelj and Ferligoj, 2005; Batagelj, Ferligoj and Doreian, 1992) is applied. Blockmodeling is an approach for reducing large, potentially incoherent networks to smaller, comprehensible and interpretable structures. In the case of direct blockmodeling, the units are relocated from one to another cluster in such a way that minimises the value of a criterion function CF (Batagelj, 1997; Doreian, Batagelj and Ferligoj, 1994). The CF must be in line with the selected equivalence. Structural equivalence is used in this study. By definition, two units are structurally equivalent if they are linked to the same others (Lorrain and White, 1971). The term "non-specified" refers to the instance when only the number of clusters is pre-specified along with the allowed block types. When structural equivalence is used, only null and complete block types are possible. On the other hand, the term "pre-specified" refers to the case when the number of clusters, the allowed block types as well as their position in the network are pre-specified.

To perform blockmodeling, the "blockmodeling" (Žiberna, 2018) package for the R programming language is used. The blockmodels obtained are compared to the ideal asymmetric core-cohesive blockmodel. The number of different types of blocks according to the ideal blockmodel structure is used as the fit function called "inconsistent blocks". For a given network, 1 or more inconsistent blocks mean different blockmodel. In the case of many generated networks (generated in the same way, including the same selected local network mechanisms and their weights) the mean number of inconsistent blocks, higher than 0.5 , indicates a high probability that the selected mechanisms and their corresponding weights generate the networks with other blockmodel type as desired (or, as compared to). The overall number of errors of the blockmodeling structure obtained vis-à-vis the ideal one is analysed by using the relative fit function described in the following section.

In order to reduce the computational burden, only 300 randomly selected $\theta$ s are considered in the current study. The mechanisms' weights are selected so that the condition $\sum \theta_{i}^{2}=1$ is satisfied. The random values are generated by firstly sampling five values from the standard normal distribution $\phi$ and then multiplying them by scalar $\theta=\phi / \sqrt{\sum \phi_{i}}$ (Marsaglia, 1972; Muller, 1959).

\subsection{Evaluating the Global Network Structure}

The first step in evaluating how close blockmodels obtained from empirical networks are to the asymmetric core-cohesive blockmodel type was described in the previous section. Although the proposed approach is sufficient for evaluating the presence of a given blockmodel type in the empirical data, it does not reflect the overall number of errors across blocks.

To this end, the value of the criterion function (CF) (Batagelj, 1997; Doreian, Batagelj and Ferligoj, 1994), defined as the total number of inconsistencies with a pre-specified blockmodel, is typically used. The value of this CF must be as low as possible. CF val- 
ues obtained for different types of blockmodels (including different numbers of clusters) are not directly comparable (e.g., increasing the number of clusters lowers the value of the $\mathrm{CF}$ ). To avoid this issue, the relative fit function (RF) can be defined. Here, the CF value obtained on an empirical network can be compared with the expected or minimal and maximal value of the empirical function obtained on many randomised networks. Simulations confirm that a higher number of iterations is needed in order to estimate the maximum value of a $\mathrm{CF}$ (in the case of a random network under the assumed blockmodel) than to estimate the expected value of the CF. Therefore, the RF is defined as

$$
R F=1-\frac{P^{m}}{\frac{1}{k} \sum_{i=1}^{k} P_{i}^{r}}
$$

where $P^{m}$ is the value of the $\mathrm{CF}$ obtained on the empirical network and $P_{i}^{r}$ is the value of the CF obtained on the $i$-th randomised network. There are $k$ randomised networks. The mean value of the $\mathrm{CF}$ in the case of random networks is estimated by simulations since it depends on many factors (e.g., the algorithm implemented for generalised blockmodeling or the density) and cannot therefore be analytically calculated. The networks used to estimate the mean $\mathrm{CF}$ value can be randomised in different ways, e.g., by preserving the degree (Rao, Jana and Bandyopadhyay, 1996) or by preserving the density of the network (Erdös and Rényi, 1959). As proposed by Boyd et al. (2006) for a core-periphery structure, the density is considered as fixed, while the distribution of the degrees can vary.

In either way, the maximum value of the RF equals 1 when there are no inconsistencies while the value of 0 indicates that the fit of the pre-specified blockmodel to empirical network is the same as it would be expected when the same pre-specified blockmodel would be applied on a randomised empirical network.

When defining the RF as described above, the RF values should be comparable across different types of blockmodels with different numbers and sizes of clusters. Interpreting RF values is the most suitable when the assumed blockmodel type can be found with the non-specified blockmodeling approach.

Observing the RF values after each of the several iterations in the algorithm used can help to evaluate the convergence of the network's global structure (i.e., when the global network structure stopped changing significantly). One way or the other is assumed to be the case when the RF values stop changing significantly (in this study, this was done by visually observing the trajectories of the mean RF values for different blockmodel types, Figure 3, Figure 5 and Figure 7). Another way of assessing the convergence of the global network structure is by observing the distribution of different types of triads since they can be used to generate the most common blockmodels (Cugmas, Ferligoj and Žiberna, 2018).

\section{Results}

The section has three parts. Different initial global network structures are assumed in each part (empty networks, networks with a cohesive blockmodel structure and networks with an asymmetric core-periphery blockmodel structure). The remaining methodology is the same for all three parts: 300 vectors containing the randomly selected mechanisms' weights are generated; 30 networks are generated for each set of the mechanisms' 
weights; each network is generated upon 32269 iterations. ${ }^{5}$ The intermediate generated networks are also analysed. Specifically, non-specified generalised blockmodeling is applied to the empirical networks with three groups assumed and null and complete blocks allowed (structural equivalence is considered). Based on this, the average number of inconsistent blocks is calculated as described in the previous sections. The ten $\theta$, generating networks with the lowest number of inconsistent blocks, are selected for each type of initial global network structure (the results for other $\theta$ s are given in Appendix 1). The networks are then generated again, based on the selected $\theta$ with an increased number of iterations up to 108694 iterations. The pre-specified blockmodeling (an asymmetric core-cohesive and asymmetric core-periphery with three groups, and a cohesive blockmodel with two groups are assumed) is applied and the RF values are calculated in order to more precisely evaluate the global network structure.

\subsection{From an Empty Network to an Asymmetric Core-Cohesive Block- model}

The mean number of inconsistent blocks along with the corresponding weights of the mechanisms in instances where the initial networks are empty networks are presented in Table 1 . Note that only the results for the first ten best $\theta$ s (according to the mean number of inconsistent blocks after 32269 iterations) are given.

The mechanisms' weights shown in Table 1 are not directly comparable. One reason for this is that the mechanisms are interdependent while the other reason is that they are not defined for absolute comparability. The highest weights are usually for the transitivity, mutuality and assortativity mechanisms. The values of the popularity mechanism are generally lower, especially when the value of the transitivity mechanism is high.

The mean number of inconsistent blocks is relatively low for the generated networks according to the mechanisms' weights given in Table 1. Yet the mean number is especially low for those networks generated according to the first five best $\theta$ s. The mean RF values ${ }^{6}$ are similar for the networks generated by the first ten sets of mechanisms' weights.

The mean RF values for the first set of mechanism weights (in Table 1) are visualised in Figure 3: the $\log x$-axis represents the number of iterations while the mean RF values are on the $y$-axis. Usually, the mean RF values, corresponding to the asymmetric core-periphery, are around zero until iteration 686. On the other hand, the mean RF values, corresponding to the asymmetric core-cohesive and cohesive blockmodel types, are increasing with the same speed (the mean values, corresponding to the cohesive blockmodel type, are usually slightly higher). This might indicate that cohesive groups are

\footnotetext{
${ }^{5}$ The intermediate number of iterations $m$, at which the global network structure is analysed, is determined as $m_{i}=m_{i-1}+m_{i-1} \cdot 1.9$, where $m_{1}=100$ and in the case of $m_{11}, \ldots, m_{15}$, the values are calculated as $m_{i}=m_{i-1}+m_{10}$. Such an approach is used since the preliminary analyses showed the global network structure changes more rapidly at a lower number of iterations. Therefore, more frequent insights into the global network structures are needed at a lower number of iterations. The values $m_{11}, \ldots, m_{15}$ are calculated differently in order to avoid a high number of iterations implied by the constant 1.9.

${ }^{6}$ For the selected sets of the mechanisms' weights (given in Table 1, Table 2 and Table 3), the RF values are distributed symmetrically around the mean RF across simulations, yet there are some networks with the extreme values of the RF. This can also be seen in Figure 3, Figure 5 and Figure 6 and in the figures given in Appendix 2.
} 
Table 1: Mean number of inconsistent blocks and the corresponding mechanisms' weights $(\theta)$ for the best ten sets of the mechanisms' weights according to the mean number of inconsistent blocks (initial is an empty network)

\begin{tabular}{|c|c|c|c|c|c|c|c|c|c|c|c|c|c|c|c|}
\hline \multirow[b]{2}{*}{ ID } & \multicolumn{5}{|c|}{$\theta$} & \multicolumn{10}{|c|}{ Number of iterations } \\
\hline & M & $\mathrm{P}$ & A & $\mathrm{T}$ & $\mathrm{O}$ & 100 & 190 & 361 & 686 & 1303 & 2478 & 4705 & 8939 & 16984 & 32269 \\
\hline 224 & 0.45 & 0.10 & 0.77 & 0.44 & -0.08 & 4.30 & 3.67 & 3.37 & 2.77 & 1.12 & 0.90 & 0.40 & 0.37 & 0.20 & 0.07 \\
\hline 226 & 0.65 & -0.04 & 0.11 & 0.71 & 0.23 & 4.20 & 3.47 & 3.00 & 2.20 & 2.23 & 1.23 & 0.43 & 0.23 & 0.10 & 0.13 \\
\hline 228 & 0.59 & 0.12 & 0.24 & 0.76 & -0.08 & 4.43 & 4.03 & 3.20 & 2.47 & 1.60 & 1.03 & 0.63 & 0.27 & 0.20 & 0.17 \\
\hline 136 & 0.41 & -0.18 & 0.37 & 0.74 & -0.34 & 4.57 & 4.30 & 3.57 & 2.83 & 1.57 & 0.80 & 0.60 & 0.33 & 0.33 & 0.20 \\
\hline 238 & 0.37 & 0.61 & 0.35 & 0.00 & 0.61 & 4.43 & 4.07 & 3.40 & 2.37 & 1.67 & 1.27 & 0.50 & 0.40 & 0.30 & 0.20 \\
\hline 153 & 0.84 & -0.14 & 0.36 & 0.32 & -0.21 & 4.23 & 4.47 & 3.50 & 2.97 & 1.90 & 1.03 & 0.53 & 0.37 & 0.33 & 0.27 \\
\hline 286 & 0.52 & 0.26 & 0.33 & 0.72 & 0.16 & 4.03 & 4.07 & 3.33 & 2.43 & 1.63 & 1.40 & 0.60 & 0.40 & 0.40 & 0.27 \\
\hline 259 & 0.51 & 0.57 & 0.60 & 0.25 & 0.02 & 4.23 & 3.93 & 3.47 & 2.60 & 1.53 & 1.47 & 0.57 & 0.43 & 0.27 & 0.33 \\
\hline 40 & -0.13 & -0.06 & 0.49 & 0.85 & 0.10 & 4.20 & 4.47 & 3.17 & 2.53 & 1.10 & 0.53 & 0.53 & 0.50 & 0.43 & 0.40 \\
\hline 57 & -0.37 & -0.33 & 0.59 & 0.63 & 0.09 & 4.80 & 4.37 & 3.47 & 2.80 & 1.50 & 0.37 & 0.43 & 0.47 & 0.40 & 0.43 \\
\hline
\end{tabular}

Note: $\mathrm{M}=$ mutuality, $\mathrm{P}=$ popularity, $\mathrm{A}=$ assortativity, $\mathrm{T}=$ transitivity, $\mathrm{O}=\mathrm{OSP}$ 
formed at very early stages before the most popular group emerges, which is not in line with Schaefer et al.'s (2010) observations about the mechanisms' strength, where they expected the most popular group would have emerged first. However, the networks are very sparse (the distribution of the generated networks' density is visualised by the boxplots in Figure 3 ) at this early stage and the mean RF values are very low (less than 0.3 ).

At between 1303 and 2476 iterations, the mean RF values for all three blockmodel types are relatively close. The common global network structure cannot easily be seen by visually observing some generated networks since they look very heterogeneous. Some generated networks are shown on Figure 4. They are drawn at different number of iterations in line with the blockmodels obtained by generalised blockmodeling (non-specified model).

Common to all networks at this number of iterations is that they become much denser. In some networks, this is expressed in the global network structures, which are close to the asymmetric core-periphery blockmodel type with the core group having half of the units. In some other cases, the global network structure, which is close to the asymmetric core-cohesive blockmodel type, appears. When this is the case, there are usually many links from the popular group to the largest cohesive group. Later, the mean RF values for the cohesive and asymmetric core-periphery largely remain constant while the mean RF values, corresponding to the asymmetric core-cohesive blockmodel type, increase. This is primarily due to the decrease in the number of errors in the null blocks.

The networks generated by considering the second and fifth $\theta$ s are outstanding (not shown in the paper). The first entails very low variability of the RF values and the second one a very high RF mean for the asymmetric core-cohesive blockmodel type. The global network structures of the networks generated by these mechanisms' weights are very close to the proposed blockmodel type. One very typical observation for most of the generated networks is that one cohesive group is usually smaller than another, while the size of the core group is less variable across the different generated networks.

\subsection{From the Asymmetric Core-Periphery to the Asymmetric Core- Cohesive Blockmodel Type}

In this section, it is assumed the initial network has an asymmetric core-periphery blockmodel (the core group consists of 8 units) with 11 or 12 randomly relocated links. The number of inconsistent blocks is low after the first 100 iterations. Since the initial network's blockmodel is asymmetric core-periphery, a low number of inconsistent blocks is expected (the asymmetric core-periphery blockmodel is part of the asymmetric corecohesive blockmodel). The number of inconsistent blocks decreases very quickly. Specifically, four $\theta$ s generate networks where the mean number of inconsistent blocks is very close to zero after the first 4705 iterations.

The mean RF values are very similar for the networks generated by considering the first best ten $\theta$ s. The main differences lie in the variability of the $R F$ values. By looking at the RF values (Figure 5) for those networks generated by considering the first $\theta$ s given in, one can notice the expected decrease of the mean $R F$ value for the asymmetric coreperiphery blockmodel type. On the other hand, the RF values for the asymmetric corecohesive and cohesive blockmodel types are increasing with the number of iterations. The 


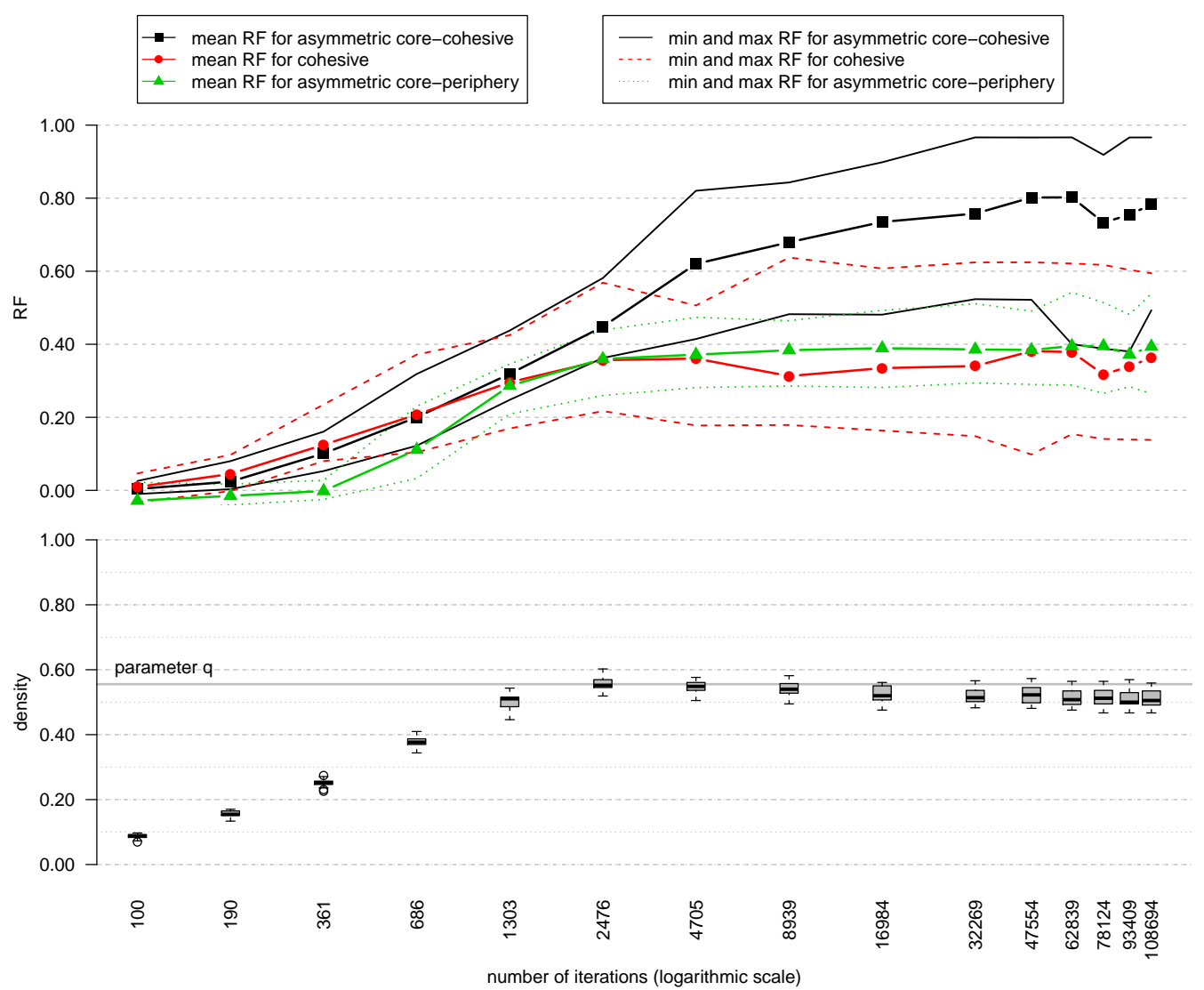

Figure 3: Mean RF for each blockmodel type visualised by lines (upper plot) and the distribution of the density visualised by boxplots (lower plot) for $\theta=\{M=0.45, P=0.10, A=$ $0.77, T=0.44, O S P=-0.88\}, q=5 / 9, d_{0}=0$

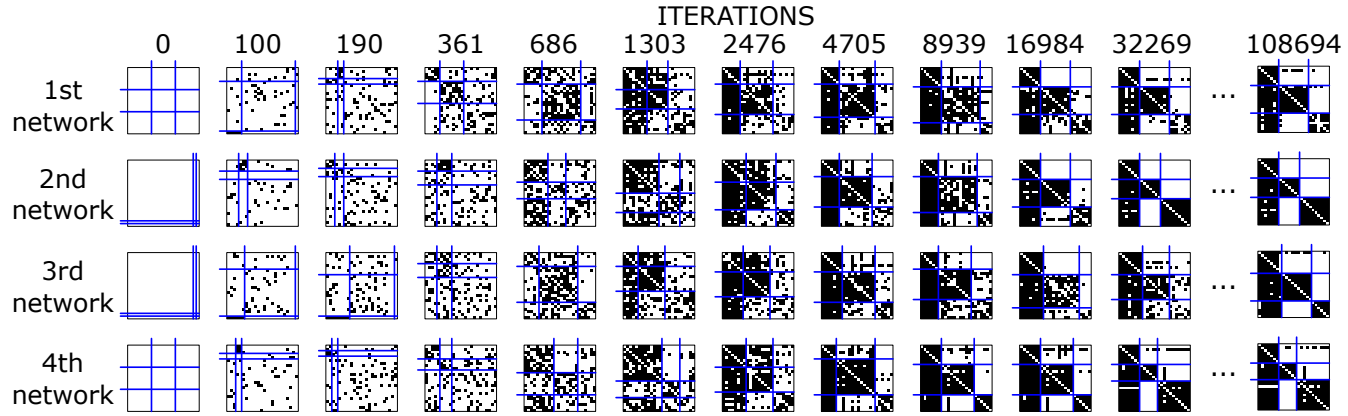

Figure 4: Some generated networks by considering $\theta=\{M=0.45, P=0.10, A=$ $0.77, T=0.44, O S P=-0.88\}$ and $q=5 / 9$; the networks are drawn in line with the blockmodels obtained (non-specified model); initial is an empty network 
Table 2: Mean number of inconsistent blocks and the corresponding mechanisms' weights $(\theta)$ for the best ten sets of the mechanisms' weights according to the mean number of inconsistent blocks (initial is the asymmetric core-periphery blockmodel type)

\begin{tabular}{|c|c|c|c|c|c|c|c|c|c|c|c|c|c|c|c|}
\hline \multirow[b]{2}{*}{ ID } & \multicolumn{5}{|c|}{$\theta$} & \multicolumn{10}{|c|}{ Number of iterations } \\
\hline & M & $\mathrm{P}$ & A & $\mathrm{T}$ & $\mathrm{O}$ & 100 & 190 & 361 & 686 & 1303 & 2478 & 4705 & 8939 & 16984 & 32269 \\
\hline 9 & 0.10 & 0.29 & 0.72 & 0.58 & 0.23 & 1.00 & 1.00 & 1.00 & 0.87 & 0.60 & 0.37 & 0.30 & 0.03 & 0.00 & 0.00 \\
\hline 40 & -0.13 & -0.06 & 0.49 & 0.85 & 0.10 & 1.43 & 1.00 & 1.00 & 0.60 & 0.53 & 0.23 & 0.03 & 0.00 & 0.00 & 0.00 \\
\hline 193 & -0.03 & 0.21 & 0.44 & 0.28 & 0.83 & 1.17 & 1.00 & 0.97 & 0.73 & 0.50 & 0.10 & 0.00 & 0.00 & 0.00 & 0.00 \\
\hline 226 & 0.65 & -0.04 & 0.11 & 0.71 & 0.23 & 1.10 & 1.07 & 0.97 & 0.77 & 0.53 & 0.30 & 0.27 & 0.07 & 0.03 & 0.00 \\
\hline 8 & 0.11 & -0.03 & 0.42 & 0.66 & 0.61 & 1.00 & 1.07 & 1.00 & 0.47 & 0.30 & 0.07 & 0.00 & 0.03 & 0.03 & 0.03 \\
\hline 57 & -0.37 & -0.33 & 0.59 & 0.63 & 0.09 & 1.63 & 1.13 & 1.00 & 0.77 & 0.30 & 0.03 & 0.03 & 0.03 & 0.03 & 0.03 \\
\hline 48 & 0.44 & 0.15 & 0.26 & 0.79 & -0.32 & 1.00 & 1.00 & 0.97 & 0.77 & 0.37 & 0.47 & 0.53 & 0.40 & 0.23 & 0.07 \\
\hline 73 & 0.01 & 0.44 & 0.43 & 0.36 & 0.70 & 1.00 & 1.00 & 1.00 & 0.87 & 0.73 & 0.47 & 0.17 & 0.10 & 0.10 & 0.10 \\
\hline 134 & -0.18 & 0.20 & 0.60 & 0.21 & 0.72 & 1.27 & 1.00 & 1.00 & 0.73 & 0.60 & 0.30 & 0.13 & 0.10 & 0.10 & 0.10 \\
\hline 202 & -0.28 & 0.48 & 0.47 & 0.15 & 0.67 & 1.83 & 1.07 & 1.13 & 0.77 & 0.77 & 0.23 & 0.33 & 0.20 & 0.10 & 0.10 \\
\hline
\end{tabular}

Note: $\mathrm{M}=$ mutuality, $\mathrm{P}=$ popularity, $\mathrm{A}=$ assortativity, $\mathrm{T}=$ transitivity, $\mathrm{O}=\mathrm{OSP}$ 
curves are parallel. This is the logical implication of the emergence of cohesive groups in the initial asymmetric core-periphery blockmodel type.

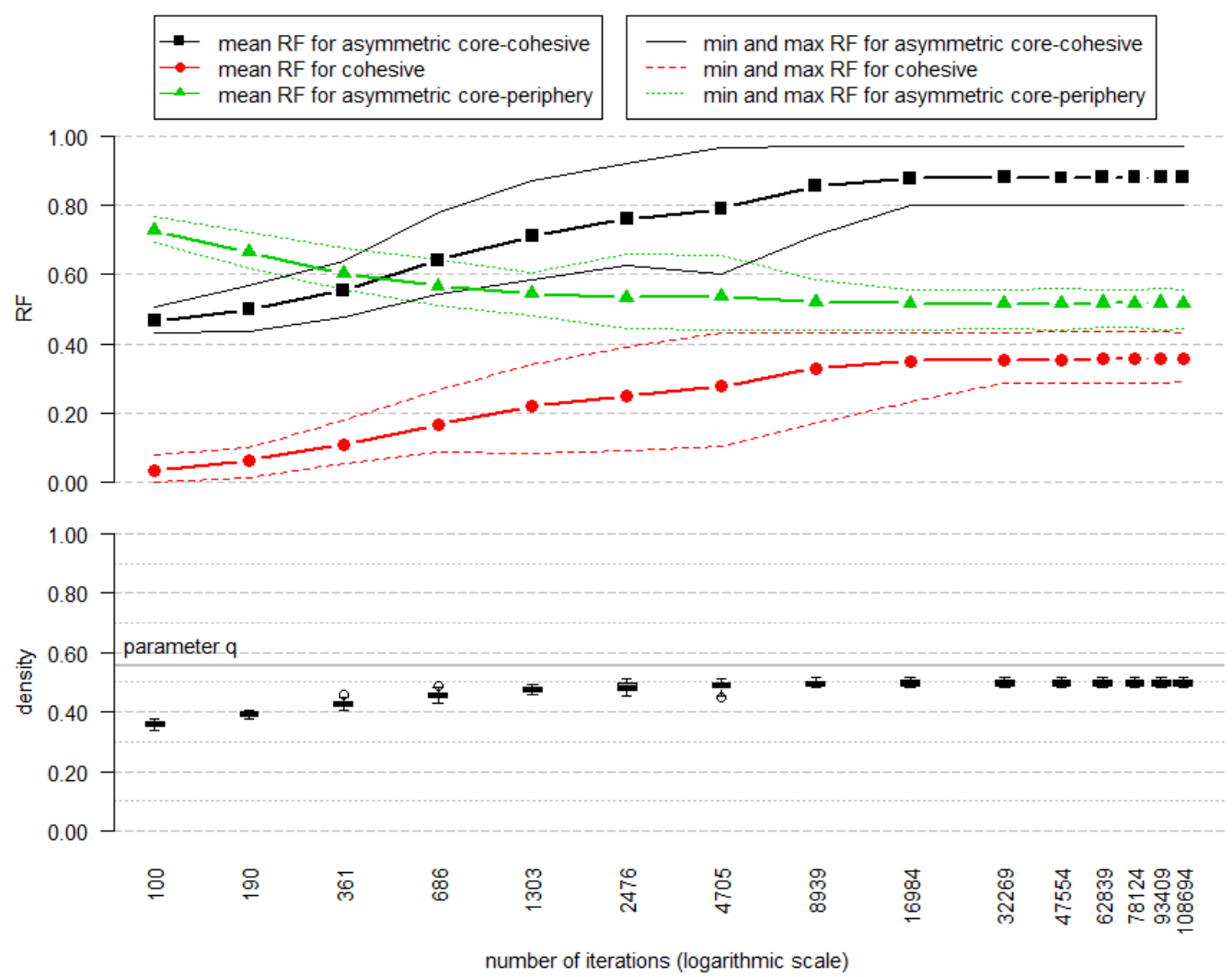

Figure 5: Mean RF for each blockmodel type visualised by lines (upper plot) and the distribution of the density visualised by boxplots (lower plot), initial are networks with an asymmetric core-periphery blockmodel (with some number errors) for $\theta=\{M=0.10, P=0.29, A=$ $0.72, T=0.58, O S P=0.23\}, q=5 / 9, d_{0}=3 / 9$

This can be confirmed by observing some randomly chosen generated networks for the first selected $\theta$ (Figure 6). The initial global network structure remains relatively unchanged after the first 100 iterations. Soon, one cohesive group emerges while the other one (which is usually smaller) becomes more prominent at a higher number of iterations.

\subsection{From the Cohesive Blockmodel to the Asymmetric Core-Cohesive Blockmodel Type}

The initial networks have a cohesive blockmodel type with 3 groups and approximately 11 randomly relocated links. Each group consists of eight units. The mean number of inconsistent blocks is 2 until 361 iterations for all best ten $\theta$ s that generate networks with the lowest mean value of the number of inconsistent blocks at the last iteration. This is expected, as explained in the previous section (the asymmetric core-periphery blockmodel and the asymmetric core-cohesive blockmodel differ in two blocks). However, the mean number of inconsistent blocks decreases faster with the asymmetric core-periphery 


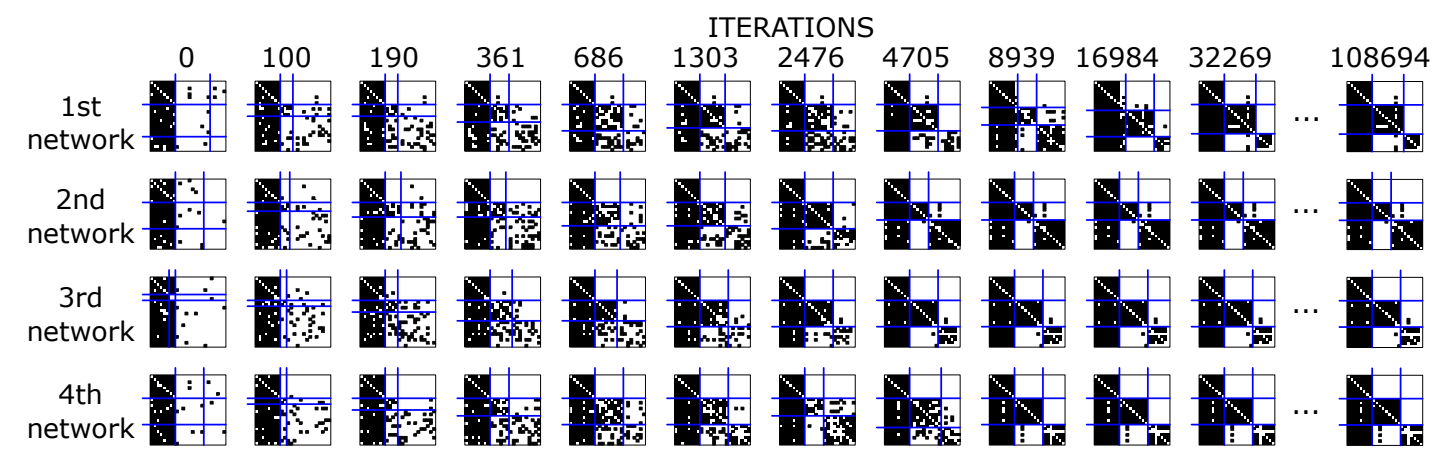

Figure 6: Some networks generated by considering $\theta=\{M=0.10, P=0.29, A=$ $0.72, T=0.58, O S P=0.23\}$ and $q=5 / 9$; the networks are drawn in line with the blockmodels obtained (non-specified model); initial blockmodel is core-periphery

blockmodel type. In addition, it seems the global network structures generally do not reach equilibrium. The number of iterations is therefore increased, when calculating the RF values, to 108694 (see Table 3).

After the first 100 iterations, the mean RF for the cohesive blockmodel type is the highest, as expected. Until 686 iterations, the values decrease while at a higher number of iterations they remain relatively stable. On the other hand, the mean RF values for the asymmetric core-periphery blockmodel type are increasing with the number of iterations - mostly between iteration 361 and 4705. This pattern is similar for the other 10 selected mechanisms' weights. The mean RF for the asymmetric core-cohesive blockmodel type is the highest after 62839 iterations.

When looking at some generated networks (Figure 8), it becomes clear that the asymmetric core-cohesive blockmodel type can be seen after 8939 iterations in most networks. Between 361 and 2476 iterations, there is no recognisable global network structure for three groups. This transition phase is common for many of the other selected $\theta$ s.

\section{Discussion on the Mechanisms}

In order to determine the best set of the mechanisms' weights that generate networks with the global structure closest to the asymmetric core-cohesive blockmodel, 300 different sets of mechanisms' weights were randomly selected. The same values were used to generate the networks for different initial global structures. Based on the number of inconsistent blocks, the ten best sets of mechanisms' weights were chosen for each initial structure. In total, only 19 different sets of mechanisms' weights were chosen.

To be more precise, when the initial network is a cohesive or empty network it is possible to generate networks with a global network structure sufficiently close to the asymmetric core-cohesive blockmodel type by considering eight different set of mechanisms' weights (see Figure 9). The asymmetric core-periphery initial network structure shares only one common set of mechanisms' weights with the cohesive initial global network structure and two sets of mechanisms' weights with the empty network as an initial network. The seven sets of mechanisms' weights, which were selected only where the global network structure of the initial networks was asymmetric core-periphery, are 


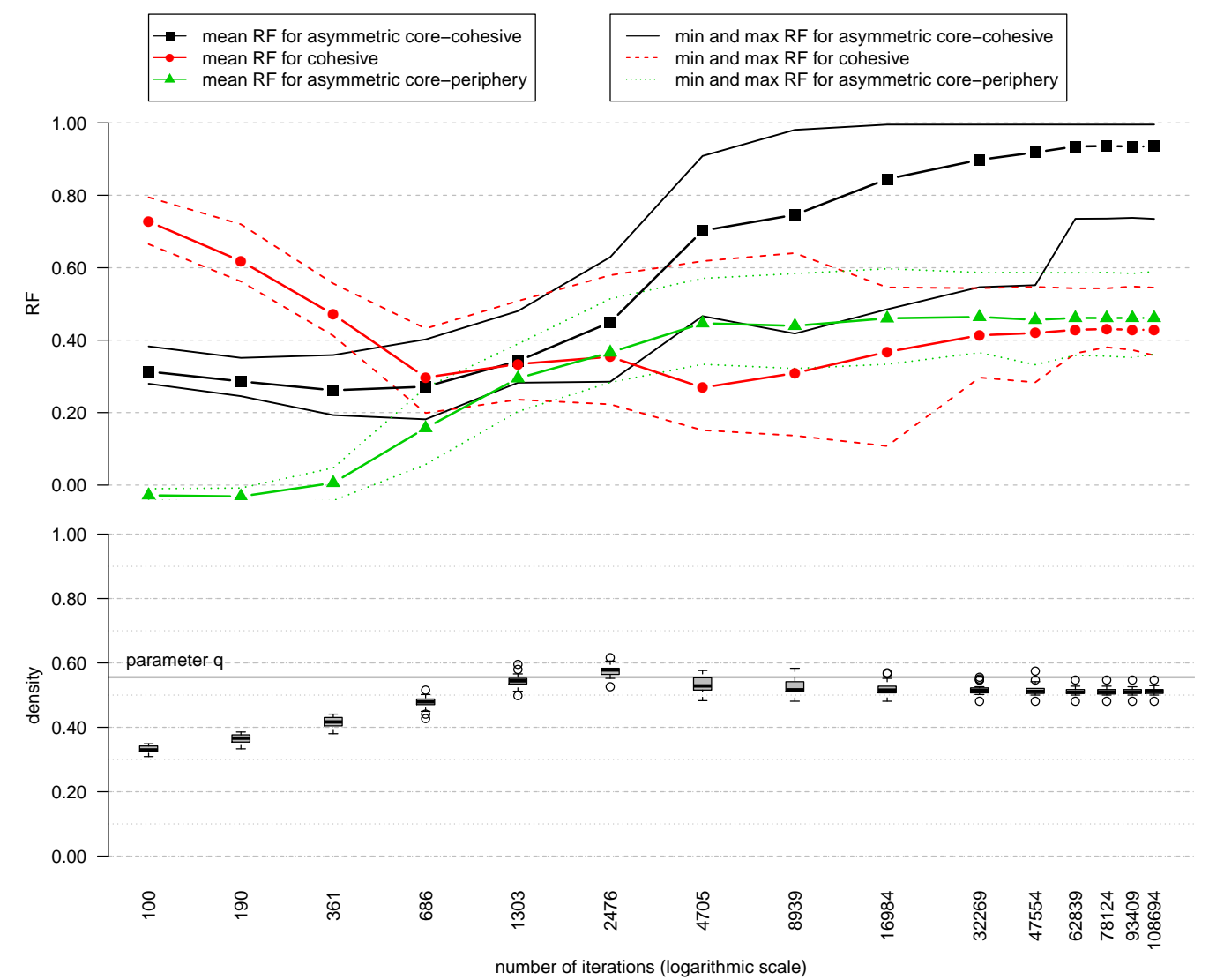

Figure 7: Mean RF for each blockmodel type visualised by lines (upper plot) and the distribution of the density visualised by boxplots (lower plot), initial are networks with a cohesive blockmodel type (with some number errors) for $\theta=\{M=0.37, P=0.61, A=0.35, T=$ $0.00, O S P=0.61\}, q=5 / 9, d_{0}=3 / 9$

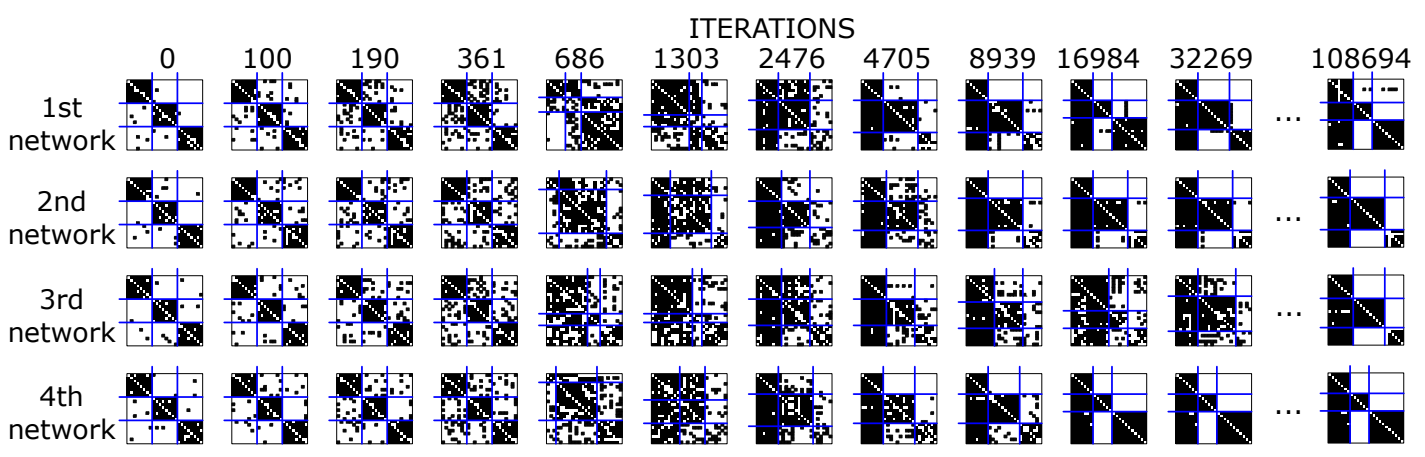

Figure 8: Some generated networks by considering $\theta=\{M=0.37, P=0.61, A=$ $0.35, T=0.00, O S P=0.61\}$ and $q=5 / 9$; the networks are drawn in line with the blockmodels obtained (non-specified model); initial blockmodel is cohesive 
Table 3: Mean number of inconsistent blocks and the corresponding mechanisms' weights $(\theta)$ for the best ten sets of the mechanisms' weights according to the mean number of inconsistent blocks (initial is the cohesive blockmodel type)

\begin{tabular}{|c|c|c|c|c|c|c|c|c|c|c|c|c|c|c|c|}
\hline \multirow[b]{2}{*}{ ID } & \multicolumn{5}{|c|}{$\theta$} & \multicolumn{10}{|c|}{ Number of iterations } \\
\hline & $\mathrm{M}$ & $\mathrm{P}$ & $\mathrm{A}$ & $\mathrm{T}$ & $\mathrm{O}$ & 100 & 190 & 361 & 686 & 1303 & 2478 & 4705 & 8939 & 16984 & 32269 \\
\hline 238 & 0.37 & 0.61 & 0.35 & 0.00 & 0.61 & 2.00 & 2.00 & 2.00 & 1.77 & 1.40 & 1.00 & 0.47 & 0.30 & 0.13 & 0.10 \\
\hline 136 & 0.41 & -0.18 & 0.37 & 0.74 & -0.34 & 2.00 & 2.00 & 2.00 & 1.63 & 0.93 & 0.67 & 0.57 & 0.17 & 0.17 & 0.17 \\
\hline 228 & 0.59 & 0.12 & 0.24 & 0.76 & -0.08 & 2.00 & 2.00 & 2.00 & 1.87 & 1.47 & 0.87 & 0.47 & 0.33 & 0.23 & 0.17 \\
\hline 224 & 0.45 & 0.10 & 0.77 & 0.44 & -0.08 & 2.00 & 2.00 & 2.00 & 1.87 & 1.00 & 0.90 & 0.37 & 0.27 & 0.23 & 0.20 \\
\hline 153 & 0.84 & -0.14 & 0.36 & 0.32 & -0.21 & 2.00 & 2.00 & 1.97 & 1.67 & 0.83 & 0.47 & 0.30 & 0.57 & 0.60 & 0.23 \\
\hline 286 & 0.52 & 0.26 & 0.33 & 0.72 & 0.16 & 2.00 & 2.00 & 2.00 & 2.00 & 1.53 & 1.30 & 0.60 & 0.33 & 0.37 & 0.27 \\
\hline 226 & 0.65 & -0.04 & 0.11 & 0.71 & 0.23 & 2.00 & 2.00 & 2.00 & 2.07 & 2.83 & 1.27 & 0.70 & 0.33 & 0.37 & 0.30 \\
\hline 259 & 0.51 & 0.57 & 0.60 & 0.25 & 0.02 & 2.00 & 2.00 & 2.00 & 1.57 & 1.10 & 1.30 & 0.50 & 0.67 & 0.53 & 0.33 \\
\hline 163 & 0.44 & 0.15 & 0.49 & 0.41 & 0.61 & 2.00 & 2.00 & 2.00 & 2.17 & 1.57 & 1.27 & 0.67 & 0.53 & 0.37 & 0.37 \\
\hline 172 & 0.28 & 0.80 & 0.17 & -0.01 & 0.51 & 2.00 & 2.00 & 2.00 & 1.90 & 1.63 & 1.50 & 1.03 & 0.83 & 0.67 & 0.50 \\
\hline
\end{tabular}

Note: $\mathrm{M}=$ mutuality, $\mathrm{P}=$ popularity, $\mathrm{A}=$ assortativity, $\mathrm{T}=$ transitivity, $\mathrm{O}=\mathrm{OSP}$ 
strongly characterised by high values on the transitivity and/or OSP mechanisms, which is expected since these promote the forming of cohesive groups while the most popular one already exists. In short, the same set of mechanisms' weights can lead to a network with the same global network structure, especially when the initial global network structure is a cohesive or empty network.

Figure 10 illustrates the associations between different weights of the five mechanisms considered. The same 19 chosen sets of the mechanisms' weights are presented as described in the previous paragraph. The sizes of the points are proportional to the mean RF values. Since most of the mean RF values are very similar (and high), the sizes of the points are similar. Different colours indicate the different global structures of the initial networks.

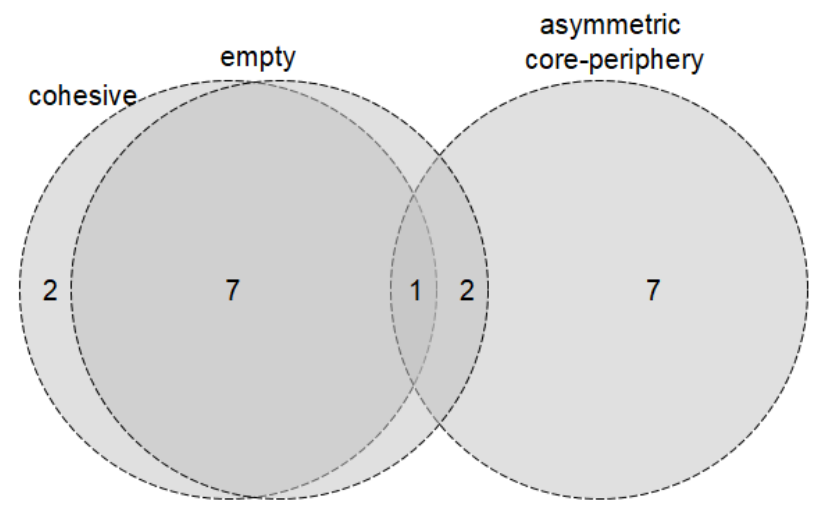

Figure 9: Number of the best common selected sets of the mechanisms' weights according to the initial networks with different global structures

A relatively strong linear negative correlation is observed between the popularity and transitivity mechanisms. This indicates that when one mechanism has a high weight, the other one becomes less important for the asymmetric core-cohesive blockmodel to appear. The transitivity mechanism is generally known, in the case of undirected networks, as being responsible for the formation of cohesive groups, yet, in the case of directed networks considered in this study, it also promotes links from the units of cohesive groups to the units of the core group. Let us assume that unit $j$ (in Figure 2, OTP) is a unit from the core group and units $i$ and $k$ are from the same cohesive group (a reciprocated or nonreciprocated link exists between them). In such a case, when the transitivity mechanism is applied a link from $i$ to $j$ would be promoted (if $i \rightarrow j$ exist), that is the link between the unit from the cohesive group to the unit from the core group. The OSP mechanism, on the other hand, is also related to the formation of the cohesive groups, but does not promote the (since it is a symmetrical characteristic) establishing links to the units of the core group. Therefore, when the OSP mechanism is strong, the popularity mechanism is usually also strong. Based on the presented principles, the correlation between the popularity mechanism and the OSP mechanism is expected to be positive.

The mutuality mechanism is considerably (negatively) correlated only with the OSP mechanism and assortativity. Since the OSP is a very symmetric mechanism, unit $j$ can be selected in the proposed algorithm in a specific iteration while, in another iteration, unit $j$ can be randomly selected. In both cases, the link from one unit to another unit would 


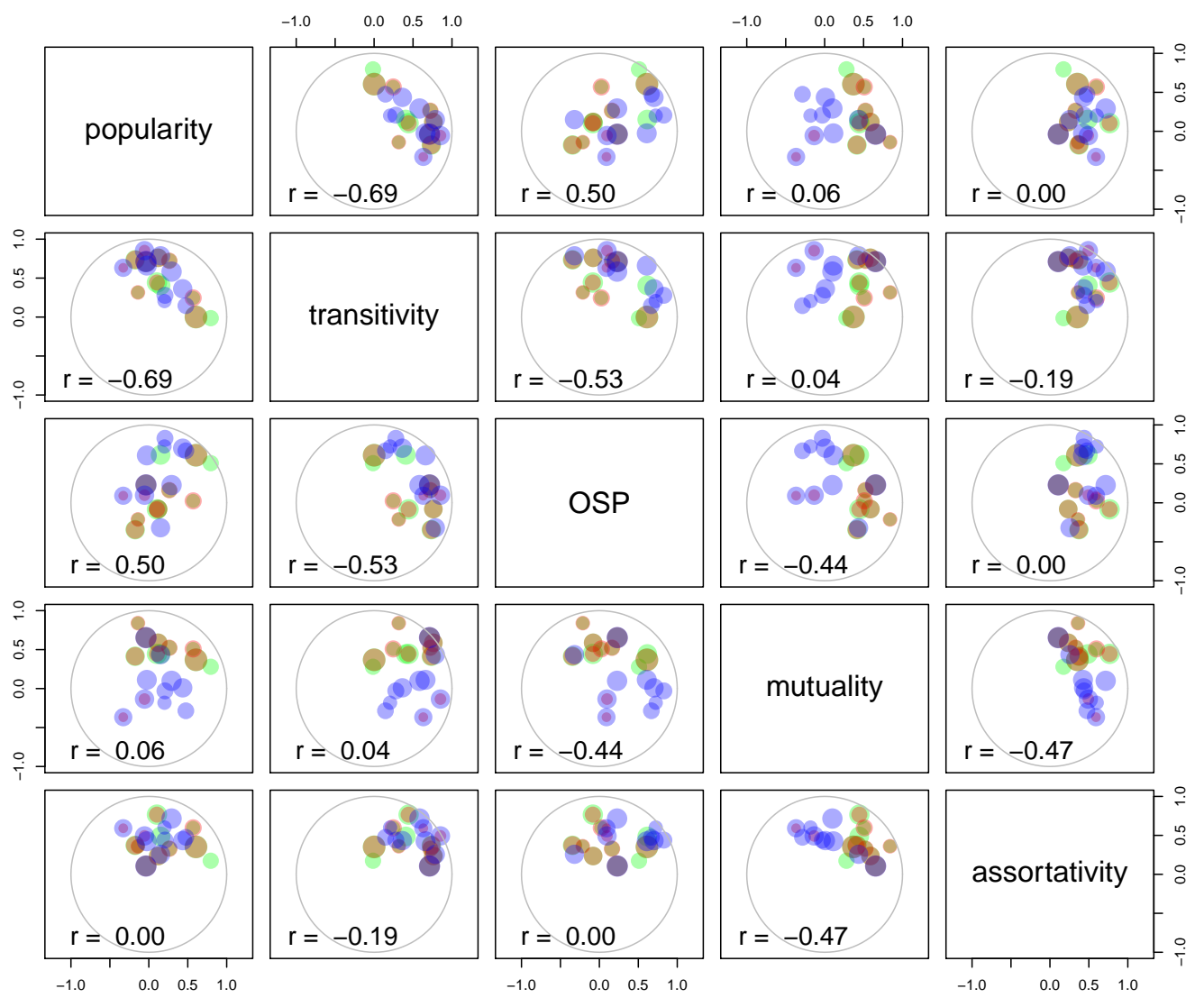

Figure 10: The associations between the mechanisms' weights (only selected sets of the mechanisms' weights are shown; the points are coloured according to the global structures of the initial networks; the sizes of the points are proportional to the mean RF values) (red = empty network; green = cohesive; blue = asymmetric core-periphery)

be promoted if they have a sufficient number of links to the same others. This eventually results in a symmetric link between units $i$ and $j$ and therefore the additional mutuality mechanism becomes less important for the asymmetric core-cohesive blockmodel type to appear.

The given interpretation is only valid when generating networks with the asymmetric core-cohesive blockmodel type. The results would be different if generating networks with different global network structures.

Depending on the initial network's global structure, one mechanism or the other might be more or less efficient in generating the asymmetric core-cohesive blockmodel type. For example, when the initial network is cohesive the popularity mechanism might be more important since the links within cohesive groups are already formed. On the contrary, when the global network structure of the initial network is asymmetric core-cohesive the shared partners' related mechanisms, i.e. the transitivity and OSP mechanisms, are important for forming the cohesive groups. 


\section{Conclusions}

The paper addresses the relationship between different local network mechanisms and global network structures. Since this is a very general aim, it is narrowed to the selected mechanisms based on the field of interest (that is preschool children networks) that can lead towards the proposed asymmetric core-cohesive blockmodel type. Understanding this relationship is necessary for studying empirically obtained networks.

The relationship between the local network mechanisms and the global network structure can be better studied when the context of the study is also considered. Therefore, the local network mechanisms are selected based on the previous studies on the network evolution of the liking, popularity and friendship networks among children in kindergarten. The selected mechanisms are: popularity (the tendency to create links to more popular ones), transitivity (the tendency to create links with those with whom a higher number of friends is shared), outgoing shared partners (the tendency to create links to those who "like the same others") and assortativity (the tendency to create links to others with the same level of popularity, i.e. in-degree).

The proposed global network structure (the asymmetric core-cohesive blockmodel) is assumed to emerge in such networks. It is a combination of two well-known blockmodel types, namely cohesive and asymmetric core-periphery.

The research question is addressed by employing the proposed algorithm, which simulates the evolution of a network based on the weights of the selected local mechanisms. Different global network structures are used for the initial networks (empty network, network with a cohesive blockmodel and network with an asymmetric core-periphery blockmodel) and 300 random sets of mechanisms' weights are generated. For each set of weights and each starting global structure, 30 networks are simulated. To evaluate the obtained global network structure, the number of inconsistent blocks, where the obtained blockmodel structure is compared to the ideal asymmetric core-cohesive blockmodel type, is considered. The proposed relative fit function is also applied for each generated network structure. Based on these two criteria the sets of mechanisms' weights that lead to the desired global network structure are evaluated for each initial network.

The major finding is that the selected five mechanisms with different mechanisms' weights can lead the networks to the asymmetric core-cohesive blockmodel. This is true for each of the considered initial global network structure. Yet, the sets of the mechanisms' weights are very similar if the initial network is empty or it has a cohesive network structure. The weights are different when the initial network has asymmetric coreperiphery structure. The findings are especially relevant as it is assumed, in this study, that the only information that units have about the other units is their position in the network.

Over the course of evolutionary process, it takes time for a blockmodel to become apparent. In the case when the asymmetric core-cohesive blockmodel is the final one, the time (i.e., number of iterations) needed to reach this blockmodel type is longer when the initial network is cohesive blockmodel, compared to other studied initial global network structures (empty network and asymmetric core-periphery blockmodel). Also, "transition" global network structures might appear in some cases. For example, when the initial network is empty, the network structure, which is close to the, for example, cohesive blockmodel, might appear after some iterations. When studding empirical networks, a researcher usually does not know at which stage of the network evolution the data were 
collected. This confirms that such studies, as the one in the proposed paper, are important for the considered specific context.

The applied methodology can be used to study the relationships between other global network structures and local network mechanisms. It is important that they are carefully chosen according to the context of the study. The proposed algorithm for generating networks could be also adjusted or extended to different assumptions (e.g., in such a way that the mechanisms' weights are considered as being variable in time or across the units or that the valued networks are generated.

\section{Acknowledgement}

The project was financially supported by the Slovenian Research Agency (www.arrs.gov.si) within the "Young Researchers" programme, research program P5-0168, the research project J7-8279 (Blockmodeling multilevel and temporal networks) and by COSTNET (CA15109). The work of Anuška Ferligoj was partly supported by the Russian Academic Excellence Project 5-100. We would like to thank the anonymous reviewers for very constructive and helpful comments and suggestions.

\section{References}

[1] Adams R. G. and Torr R. (1998): Factors underlying the structure of older adult friendship networks. Social Networks, 20(1), 51-61.

[2] Batagelj V. (1997): Notes on blockmodeling. Social Networks, 19(2), 143-155.

[3] Batagelj V., Ferligoj A. and Doreian P. (1992): Direct and indirect methods for structural equivalence. Social Networks, 14(1), 63-90.

[4] Block P. (2015): Reciprocity, transitivity, and the mysterious three-cycle. Social Networks, 40(1), 163-173.

[5] Boyd J. P., Fitzgerald W. J. and Beck R. J. (2006): Computing core/periphery structures and permutation tests for social relations data. Social Networks, 2(28), 165178.

[6] Brown B. B., Eicher S. A. and Petrie S. (1986): The importance of peer group ("crowd") affiliation in adolescence. Journal of Adolescence, 9(1), 73-96.

[7] Bukowski W. M., Pizzamiglio T. M., Newcomb A. F. and Hoza B. (1996): Popularity as an affordance for friendship: The link between group and dyadic experience. Social Development, 5(2), 189-202.

[8] Cillessen A. H. and Rose A. J. (2005): Understanding popularity in the peer system. Current Directions in Psychological Science, 14(2), 102-105.

[9] Cugmas M., Ferligoj A. and Žiberna A. (2018): Generating global network structures by triad types. PLoS One, 5(13), e0197514. 
[10] Daniel J. R., Santos A. J., Peceguina I. and Vaughn B. E. (2013): Exponential random graph models of preschool affiliative networks. Social Networks, 35(1), 25-30.

[11] Doreian P., Batagelj V. and Ferligoj A. (1994): Partitioning networks based on generalized concepts of equivalence. Journal of Mathematical Sociology, 19(1), 1-27.

[12] Doreian P., Batagelj V. and Ferligoj A. (2005): Generalized blockmodeling. Cambridge: University Press.

[13] Engle J. M., McElwain N. L. and Lasky N. (2011): Presence and quality of kindergarten children's friendships: Concurrent and longitudinal associations with child adjustment in the early school years. Infant and Child Development, 20(4), 365386.

[14] Erdös P. and Rényi A. (1959): On random graphs, I. Publicationes Mathematicae (Debrecen), 6(1), 290-297.

[15] Hartup W. W. (1993): Adolescents and their friends. New Directions for Child and Adolescent Development, 1993(60), 3-22.

[16] Hartup W. W. and Stevens N. (1997): Friendships and adaptation in the life course. Psychological Bulletin, 121(3), 355-370.

[17] Hunter D. R., Goodreau S. M. and Handcock M. S. (2013): ergm. userterms: A Template Package for Extending statnet. Journal of Statistical Software, 52(2), 2.

[18] Jansson I. (1997): Clique structure in school class data. Social Networks, 19(3), 285-301.

[19] Jansson I. (2000): Popularity structure in friendship networks. Social Networks, 21(4), 339-359.

[20] Johnson J. C., Ironsmith M., Whitcher A. L., Poteat G. M., Snow C. W. and Mumford S. (1997): The development of social networks in preschool children. Early Education and Development, 8(4), 389-405.

[21] Kerns K. A. (2000): Types of preschool friendships. Personal Relationships, 7(3), 311-324.

[22] LaFontana K. M. and Cillessen A. H. (2002): Children's perceptions of popular and unpopular peers: A multimethod assessment. Developmental Psychology, 38(5), 635.

[23] Lorrain F., and White H. C. (1971): Structural equivalence of individuals in social networks. The Journal of Mathematical Sociology, 1(1), 49-80.

[24] Marsaglia G. (1972): Choosing a point from the surface of a sphere. The Annals of Mathematical Statistics, 43(2), 645-646.

[25] Muller M. E. (1959): A note on a method for generating points uniformly on ndimensional spheres. Communications of the ACM, 2(4), 19-20. 
[26] Proulx M. F. and Poulin F. (2013): Stability and change in kindergartners' friendships: Examination of links with social functioning. Social Development, 22(1), $111-125$.

[27] Rao A. R., Jana R. and Bandyopadhyay S. (1996): A Markov chain Monte Carlo method for generating random $(0,1)$-matrices with given marginals. Sankhya: The Indian Journal of Statistics, 58(2), 225-242.

[28] Robins G., Pattison P., Kalish Y. and Lusher D. (2007): An introduction to exponential random graph ( $\left.\mathrm{p}^{*}\right)$ models for social networks. Social Networks, 29(2), 173-191.

[29] Robins G., Pattison P. and Wang P. (2009): Closure, connectivity and degree distribution: Exponential random graph $\left(\mathrm{p}^{*}\right)$ models for directed social networks. Social Networks, 31(1), 105-117.

[30] Schaefer D. R., Light J. M., Febes R. A., Hanish L. D. and Martin C. L. (2010): Fundamental principles of network formation among preschool children. Social Networks, 32(1), 61-71.

[31] Snijders T. A. (1996): Stochastic actor-oriented models for network change. Journal of Mathematical Sociology, 21(1-2), 149-172.

[32] Snijders T. A., Pattison P. E., Robins G. L. and Handcock M. S. (2006): New specifications for exponential random graph models. Sociological Methodology, 36(1), 99-153.

[33] Snijders T. A., Van de Bunt G. G. and Steglich C. E. (2010): Introduction to stochastic actor-based models for network dynamics. Social Networks, 32(1), 44-60.

[34] Snyder J., West L., Stockemer V., Gibbons S. and Almquist-Parks L. (1996): A social learning model of peer choice in the natural environment. Journal of Applied Developmental Psychology, 17(2), 215-237.

[35] Toivonen R., Kovanen L., Kivelä M., Onnela J. P., Saramäki J. and Kaski K. (2009): A comparative study of social network models: Network evolution models and nodal attribute models. Social Networks, 31(4), 240-254.

[36] Zeggelink E. (1994): Dynamics of structure: An individual oriented approach. Social Networks, 16(4), 295-333.

[37] Žiberna A. (2018): Blockmodeling 0.3.1: An R package for Generalized and classical blockmodeling of valued networks. Retrieved 19. 7. 2018 from https://cran.rproject.org/web/packages/blockmodeling/index.html. 